\title{
Special Issue on Smart Production
}

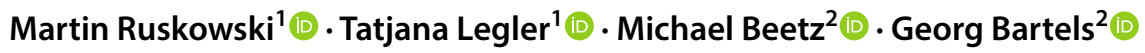

๑ Gesellschaft für Informatik e.V. and Springer-Verlag GmbH Germany, part of Springer Nature 2019

\section{Research Overview}

Today a lot of discussion is held about the positions and roles of AI research in Europe compared to the US and China. Whereas US companies focus on consumer driven applications like social media and search machines and China is predominant in state driven AI projects like face recognition, there seems to be a consensus that the business to business area and especially production is the strength of Europe and thus should be the major application field for European AI.

Production is strongly coupled with automation and the latest step in the industrial development of the past centuries is Industrie 4.0 as an output of the German high-tech strategy. In the past years many people considered Industrie 4.0 to be a technological challenge only. But it became apparent that the replacement of one technology with another does not lead to new business models nor cost reduction.

Instead of this, Industrie 4.0 must be discussed on a more abstract level. From the very beginning the use of AI methods was a part of the Industrie 4.0 idea. But AI does not mean more sophisticated automation. On the contrary, in some applications we have already reached a peak of automation level, since highly automated systems tend to be less resilient than systems with human workers. Not only Elon Musk made the observation that "humans are underrated"

Martin Ruskowski

martin.ruskowski@dfki.de

Tatjana Legler

tatjana.legler@dfki.de

Michael Beetz

beetz@cs.uni-bremen.de

Georg Bartels

georg.bartels@cs.uni-bremen.de

1 Deutsches Forschungszentrum für Künstliche Intelligenz (DFKI), Trippstadter Straße 122, 67663 Kaiserslautern, Germany

2 Institute for Artificial Intelligence (IAI), University of Bremen, Am Fallturm 1, 28359 Bremen, Germany in a famous tweet when Tesla was struggling with setting up the Model 3 production [22].

Other than to optimize automation processes asymptotically, Industrie 4.0 has to address completely other fields in production. Today most inefficiencies occur (and most money is lost) in the non-productive processes within production, i.e. the transport of data, broken data flows, duplicate entry of data, manual reprogramming of machines etc.

AI must be used to build intelligent machines in a very special way. We need autonomous machines in a way, that all information needed to operate the machine is included in it. As of today the interface of a machine to a higher level supervisory control mainly consists of binary switches and corresponding parameters. The use of the machine (i.e. the programming of the interface on the supervisory control side) needs a lot of internal knowledge about what bits to set at which step and what to expect as the answer from the machine. The introduction of field-bus communication has only replaced the former cables between machines - the communication concept itself usually is still the same as in early days of electro-mechanical systems.

Opposed to this, intelligent machines have to behave in a form of a multi-agent system. The intelligence of a multiagent system does not result from a sophisticated intelligence inside the single machine. The whole system consisting of autonomous machines will behave in an intelligent way although the single machine has a limited scope.

Many people still associate $\mathrm{AI}$ in production mainly with robotics as intelligent machines. But when looking into the details there are far more fields of application. Some of the main areas are among others:

- Robotics

- Visual inspection

- Quality control

- Data analytics

- Predictive maintenance

- Production planning

- Multi agent systems

- Human machine interaction 


\subsection{Al from the Engineering Point of View}

Historically, AI is seen as a computer science technology. When it comes to production we meet the physical world with all its implications and challenges. For an engineer it is interesting to look at AI from a different angle.

Humans always act in a closed loop-we collect input data from our environment, try to understand it, make decisions based on this understanding and act according to these decisions. When we compare this behavior to a technical control loop we find similarities such as sensors, state observers, controllers and actuators. Thus from an engineering point of view AI can be considered as closed loop control in a very generalized way.

This leads to a significant observation-in a physical environment AI incorporates through an application. Neural networks, decision trees, automated decision making systems, augmented and virtual reality and many other technologies which usually are considered to be AI are merely methods to achieve $\mathrm{AI}-$ or more specifically machines behaving in way we consider to appear intelligent.

The analogy to closed loop control provides some hints of the limitations for AI based systems. Properties like observability and controllability are well known in engineering. Observability describes the ability to reconstruct the internal behavior of a system purely on the observation of its outputs. On the other side, controllability is important for the ability to manipulate all parts of an existing system in a desired way just from the system inputs. AI in a closed loop can only be successful if a system has both properties. Even more important is the stability of a closed loop system, since it can be difficult to design a stable control loop even for simple systems. The proper design of an AI based feedback has completely different requirements for stability than conventional systems, thus requiring significant future research.

AI applications can be divided into three main areas: The "visible" ones related to manipulation tasks and robots, those related to the mere "hidden" tasks of production planning and inter-machine communication in cyberphysical production system and finally the field of human machine interaction and new user interfaces.

\subsection{Cyber-Physical Production Systems}

Since the introduction of the term Industrie 4.0 in 2011 [16] research has procured itself with the question how to achieve an adaptive, self-configuring and therefore flexible production. One of the key components to achieving that concept are cyber-physical production systems
(CPPS) [13]. They make use of sensors and actuators to capture and influence the physical world and are interconnected via local networks or the internet. Since communication no longer has to be hierarchical, there are tendencies away from the classic automation pyramid towards a decentralized, partially self-organizing network [15]. Due to the often critical role of the units, fault tolerance and the ability to respond and adapt to a disruptive event is still a major research topic [7].

Production systems are usually controlled by programmable logic controllers (PLC). Their programming languages had been derived from electrical circuitry and wiring [24]. Since the 1980s fieldbuses have been used to connect PLC based automation systems. Unlike to computer science, where the development converged into TCP/IP as the primary communication technology, automation stuck with a huge number of different and incompatible fieldbuses [33]. But one thing is common to theses fieldbuses: They merely replace discrete wiring with a single cable but do not change the connection principle. Whereas in computer science sophisticated protocols and client/server based communication has been state-of-the-art for a long time a fieldbus mainly emulates digital and analogue signals on both sides of the connection [8].

One dependency can be considered as crucial: Automation of machines relies on deterministic real-time behavior $[18,34]$. Variable runtimes of tasks and unpredictable communication delays are not acceptable. But real-time behavior must not be confused with high CPU power, as it is mainly related to the architecture of the operating system [35]. Well known desktop operating systems like Linux and Windows are not suitable for real-time control and dedicated real-time operating systems (RTOS) are required instead. This is one of the main paradigms for Edge computing in automation [9].

When defined reaction times are needed, a deterministic transfer of simple signals is very robust and reliable. Everybody who has developed an embedded real-time software also knows about the problems in debugging a complex multitasking system. In contrast to this, the simplicity of PLC programming and the popular IEC61131-3 programming languages along with today's sophisticated engineering systems make real-time programming more accessible and easier to understand [14].

Nonetheless we encounter a drawback of this structure when it comes to cyber-physical productions systems. Whenever the communication is based on simple binary signals the communication partners need an internal knowledge of each others behavior. A command is sent via setting a bit and the reply received by a different bit and flow control is mainly performed with simple state machines [31]. Usually several machines are combined to form a cluster of devices, a production cell. Often there are real-time dependencies 
between the devices, i.e. between a machine tool, a handling robot and a connecting PLC. Motions must be synchronized, switch times coordinated and many more conditions met [37]. We often find configurations without an explicit master functionality, merely the state control of the whole production cell is distributed among the individual machines.

The step towards smart production requires a new approach to the interfacing of production cells. It is necessary to encapsulate all functions of the cell so there is no need for real-time communication to the outside. All realtime dependencies shall be handled within the cell itself. The resulting entity can then be called a cyber-physical production module (CPPM) [27]. A necessary step is the definition of a master controller interfacing to the individual machines providing a service-oriented interface to the orchestration layer of the cyber-physical production system. The resulting system architecture shows a certain analogy to multi-agent system [19].

For the broad acceptance of cyber-physical production systems a standardization of the architecture, the interfaces and the orchestration is crucial. A number of contributions in this edition show possible approaches for these interfaces and for the orchestration layer paving the path towards real smart production.

\subsection{Al-Based Robotics}

Similarly to the engineering perspective, white papers and road-maps for AI-based robotics identify smart production as one of its key target domains [2, 30]. Indeed, economic pressure, the desire to bring back production to high-wage countries, as well as supporting an aging work-force motivate intense research activities towards more intelligent robotic agents on the shop floor. Important research trends that currently experience massive research interest include cloud robotics, easy programming through imitation learning, accomplishing complex manufacturing tasks without the need for fixtures, digital twin representations of factories and manufacturing processes, adaptable manipulation solutions, object perception in unstructured environments, and machine learning to learn grasp poses, failure monitors, object recognition.

The current technological wave in $\mathrm{AI}$ is to a large extend driven by automated machine learning technologies, in particular deep learning. Given massive amounts of annotated training data, supervised machine learning techniques have been successfully applied to real-world perception tasks and even simple manipulation tasks such as bin picking and fetching a large variety of objects $[6,20,28]$. These technologies enable developers to implement high-performance perception and action capabilities with reasonable programming efforts.
Another way of easing the programming of robots is the increased application of imitation learning to assembly and other manipulation tasks. Imitation learning can be seen as a form of programming by demonstration [5], where the learning system uses deeper models of actions, including intentions and structured motion models. [26] A more recent trend is to realize imitation learning methods through simulation-based virtual reality environments instead of being limited to vision-based observation data. This is a promising approach because by accessing the underlying data structures of the simulation engines one can often generate accurately annotated learning data that constitute ground truth and would otherwise be hard to obtain [12].

Another important trend is knowledge-based robot programming [32]. This approach is in particular promoted by Gil Prat who states that: "Robots are already making large strides in their abilities, but as the generalizable knowledge representation problem is addressed, the growth of robot capabilities will begin in earnest, and it will likely be explosive." [25] For many years, the application of knowledgebased programming techniques have been hindered by knowledge representation techniques being too abstract. Recently, new techniques have been proposed that represent symbolic knowledge at geometric level which is necessary for properly parameterizing robot motions for accomplishing manipulation tasks and avoid undesired side effects [4].

Another approach to make the programming of so many manipulation application feasible is to crowd source the programming tasks. A major barrier in current robot programming is that robots are typically programmed for an individual combination of tasks, robots, and environments. Currently, there is little re-use of code from one application to another one. To surpass this barrier, cloud robotics has proposed that developers provide code pieces and computation services in more general forms such that they can be reused by others. This approach was pioneered in the EU FP7 project RoboEarth [36], and further pushed in Ken Goldberg's initiative for cloud robotics [17]. Today, we see that high-tech IT companies, including Amazon, Google, and Microsoft, are all proposing their own cloud platforms ${ }^{123}$.

One of the main issues that slow down the realization of new production processes in the factory floor is the need for designing and creating fixtures that make the robots' manipulation actions reliable and fast. Removing the need for such fixtures requires to have better and more flexible hand-eye coordination, as well as higher single arm and dual-arm manipulation capabilities [1,21].

\footnotetext{
1 https://aws.amazon.com/robomaker/

2 https://cloud.google.com/cloud-robotics/

${ }^{3}$ https://azure.microsoft.com/en-us/
} 
Another research direction is the development of manipulation robots that assist humans in their manipulation tasks. Such robots have particular high demands on their cognitive capabilities [3]. This is because they do not only have to plan and execute their own actions but rather understand what their human co-workers need in terms support. These robots are particularly important for manipulation tasks that are potentially hazardous or ergonomically unhealthy. As these robots share workspaces with human co-workers they need to guarantee the safety of the humans [10, 11]. Examples of particularly expressive and powerful cognitive capabilities for such robots that are currently under research include the learning of human preference models and simulation-based mechanisms for perceptive taking [23, 29].

This special issue of KI presents several of the leading developments that will help push AI-based robotics along the path outlined by public road-maps and economic demand.

\section{About the Special Issue}

Cyber-physical systems and AI-based robots are increasingly important because a growing number of industrial applications has to flexibly change or at least customize its production relatively often. Hence, supporting frequent adaptations without significant additional investment costs has become a requirement. As a result, more intelligence is required in the actual production processes, be it human or artificial in nature. As there are plenty of decision making tasks that either humans or machines excel at, economically sound solutions typically require a combination of both.

Regarding cyber-physical systems, this special issue of Künstliche Intelligenz (KI) illustrates

- a framework to enable flexible production orchestration,

- explainability of predictions in the industrial environment, and

- a service-based architecture approach that encapsulates production steps into reusable services.

With regards to AI-based robots, this special issue of KI presents examples of leading-edge developments, including

- robots that autonomously perform fetch-and-place tasks to deliver goods in warehouses and on shop floors,

- robot programming approaches using human demonstrations and background knowledge,

- reasoning about Impedance control for manipulation actions with significant contacts,

- knowledge representation and reasoning for robots that safely interact with humans in shared workspaces, and
- increasing the visual intelligence of robots such that they can perform manipulation actions on changing objects.

This special issue of KI presents challenges as well as solutions for smart production using AI technology. As such, it presents cyber-physical systems and AI-based robots not only as enabling technologies for factories that are more flexible and efficient, but also for assisting humans in production. Thus, AI technologies present opportunities to create workspaces for humans instead of demanding humans for workspaces. Furthermore, this issue illustrates that for a beneficial use of AI the necessary infrastructure must be provided. It also highlights the need for novel communication protocols and architectures, as well as production systems.

\section{Content}

\subsection{Technical Contributions}

- A Semantic-based Method for Teaching Industrial Robots New Tasks

Karinne Ramirez-Amaro, Emmanuel Dean-Leon, Florian Bergner, and Gordon Cheng

- Episodic Memories for Safety-Aware Robots-Knowledge Representation and Reasoning for Robots that Safely Interact with Human Co-Workers

Georg Bartels, Daniel Beßler, and Michael Beetz

- A Jumpstart Framework for Semantically Enhanced OPC-UA

Badarinath Katti, Christiane Plociennik, and Michael Schweitzer

- Catering to real-time requirements of cloud-connected mobile manipulators

Julian-Benedikt Scholle

- Plug, Plan and Produce as Enabler for easy Workcell Setup and Collaborative Robot Programming in Smart Factories

Michael Wojtynek, Jochen Jakob Steil, and Sebastian Wrede

\subsection{Project Reports}

- A Service-Based Production Ecosystem Architecture for Industrie 4.0

Thomas Kuhn, Siwara Sadikow, and Pablo Antonino

\subsection{Al Transfer}

- Vision-based solutions for manipulation and navigation applied to object picking and distribution

Máximo A. Roa-Garzó, Elena F. Gambaro, Monika Florek-Jasinska, Felix Endres, Felix Ruess, Raphael 
Schaller, Christian Emmerich, Korbinian Muenster, and Michael Suppa

- Towards Explainable Process Predictions for Industry 4.0 in the DFKI-Smart-Lego-Factory

Jana-Rebecca Rehse, Nijat Mehdiyev and Peter Fettke

\subsection{Interviews}

- Perception-guided Mobile Manipulation Robots for Automation of Warehouse Logistics-Interview with Dr. Moritz Tenorth, CTO of the Startup Magazino Georg Bartels and Michael Beetz

- From Research to Market: Building the Perception Systems for the Next Generation of Industrial Robots-Interview with Dr. Michael Suppa, CEO and Founder of the Startup Roboception

Georg Bartels and Michael Beetz

\subsection{Doctoral Dissertations}

- On Cognitive Reasoning for Compliant Manipulation Tasks in Smart Production Environments

Daniel Leidner

\section{Service}

\subsection{Conferences and Workshops}

- IEEE International Conference on Robotics and Automation (ICRA), https://www.icra2019.org

- IEEE/RSJ International Conference on Intelligent Robots and Systems (IROS), https://www.iros2019.org

- IEEE-RAS International Conference on Humanoid Robots (Humanoids), http://humanoids2019.loria.fr

- International Conference on Advanced Robotics (ICAR), http://www.icar2019.org

- IEEE International Conference on Simulation, Modeling, and Programming for Autonomous Robots (SIMPAR), https://simpar.uqcloud.net

- Robotics: Science and Systems (RSS), http://www.roboticsconference.org

- Flexible Automation and Intelligent Manufacturing (FAIM), https://faimconference.com

- IFAC Symposium on Mechatronic Systems (MECHATRONICS 2019), http://www.mechatronicsnolcos2019.org

- IEEE Conference on Emerging Technologies and Factory Automation (ETFA), http://www.etfa2019.org

- IEEE International Conference on Industrial Informatics (INDIN), https://www.indin2019.org/

\subsection{Journals}

- IEEE Robotics Automation Magazine (RAM), https://www.ieee-ras.org/publications/ram

- IEEE Robotics and Automation Letters (RA-L), https://www.ieee-ras.org/publications/ra-1

- Robotics and Autonomous Systems (RAS), https://www.journals.elsevier.com/robotics-and-auton omous-systems

- Intelligent Service Robotics, https://www.springer.com/ engineering/control/journal/11370

- Journal of Industrial Information Integration, https://www.journals.elsevier.com/journal-of-indus trial-information-integration

- Procedia CIRP, The International Academy for Production Engineering https://www.journals.elsevier.com/ procedia-cirp

Acknowledgements We gratefully acknowledge that this article was partially funded by the Deutsche Forschungsgemeinschaft (DFG) through the Collaborative Research Center 1320 EASE.

\section{References}

1. Smith C, Karayiannidis Y, Nalpantidis L, Gratal X, Qi P, Dv Dimarogonas, Kragic D (2012) Dual arm manipulation-a survey. Robot Autonom Syst 60(10):1340-1353. https://doi.org/10.1016/j. robot.2012.07.005

2. A roadmap for us robotics; from internet to robotics; 2016 edition. 2016. https://cra.org/ccc/wp-content/uploads/sites/2/2016/11/ roadmap3-final-rs-1.pdf. Accessed 19 Apr 2019

3. Ajoudani A, Zanchettin AM, Ivaldi S, Albu-Schäffer A, Kosuge K, Khatib O (2018) Progress and prospects of the human-robot collaboration. Auton Rob 2018:1-19

4. Beetz M, Beßler D, Haidu A, Pomarlan M, Bozcuoglu AK, Bartels G (2018) Knowrob 2.0-a 2nd generation knowledge processing framework for cognition-enabled robotic agents. In: International conference on robotics and automation (ICRA). Brisbane, Australia

5. Billard A, Calinon S, Dillmann R, Schaal S (2008) Robot programming by demonstration. Springer handbook of robotics, pp 1371-1394

6. Correll N, Bekris KE, Berenson D, Brock O, Causo A, Hauser K, Okada K, Rodriguez A, Romano JM, Wurman PR (2018) Analysis and observations from the first amazon picking challenge. IEEE Trans Autom Sci Eng 15(1):172-188

7. Flammini F (2019) Resilience of cyber-physical systems. Springer, Cham. https://doi.org/10.1007/978-3-319-95597-1

8. Galloway B, Hancke GP (2013) Introduction to industrial control networks. IEEE Commun Surv Tutor 15(2):860-880. https://doi. org/10.1109/SURV.2012.071812.00124

9. Gezer V, Um J, Ruskowski M (2018) An introduction to edge computing and a real-time capable server architecture. Int J Adv Intell Syst IARIA 11(1\&2):105-114 
10. Haddadin S (2015) Physical safety in robotics. Formal modeling and verification of cyber-physical systems. Springer, Berlin, pp 249-271

11. Haddadin S, Croft E (2016) Physical human-robot interaction. Springer handbook of robotics. Springer, Berlin, pp 1835-1874

12. Haidu A, Beetz M (2019) Automated models of human everyday activity based on game and virtual reality technology. In: International conference on robotics and automation (ICRA). Montreal, Canada

13. Hennecke A, Ruskowski M (2018) Design of a flexible robot cell demonstrator based on cpps concepts and technologies. In: 2018 IEEE industrial cyber-physical systems (ICPS). IEEE, pp 534-539. https://doi.org/10.1109/ICPHYS.2018.8390762

14. John KH, Tiegelkamp M (2010) State-of-the-art plc configuration. In: John KH (ed) IEC 61131-3. Springer, New York, pp 233-248. https://doi.org/10.1007/978-3-642-12015-2_6

15. Kaestner F, Kuschnerus D, Spiegel C, Janssen B, Huebner M (2018) Design of an efficient communication architecture for cyber-physical production systems. In: Vogel-Heuser B et al (eds) 2018 IEEE 14th international conference on automation science and engineering (CASE). IEEE, Piscataway, pp 829-835. https:// doi.org/10.1109/COASE.2018.8560563

16. Kagermann H, Lukas WD, Wahlster W (2011) Industrie 4.0: Mit dem Internet der Dinge auf dem Weg zur 4. industriellen Revolution. VDI Nachrichten

17. Kehoe B, Patil S, Abbeel P, Goldberg K (2015) A survey of research on cloud robotics and automation. IEEE Trans Autom Sci Eng 12(2):398-409

18. Konieczek B, Rethfeldt M, Golatowski F, Timmermann D (2016) A distributed time server for the real-time extension of coap. 2016 IEEE 19th international symposium on real-time distributed computing-ISORC 2016. IEEE, Piscataway, pp 84-91. https://doi. org/10.1109/ISORC.2016.21

19. Leitao P, Karnouskos S, Ribeiro L, Lee J, Strasser T, Colombo AW (2016) Smart agents in industrial cyber-physical systems. Proc IEEE 104(5):1086-1101. https://doi.org/10.1109/JPROC .2016 .2521931

20. Levine S, Pastor P, Krizhevsky A, Ibarz J, Quillen D (2018) Learning hand-eye coordination for robotic grasping with deep learning and large-scale data collection. Int J Robot Res 37(4-5):421-436

21. Maycock J, Dornbusch D, Elbrechter C, Haschke R, Schack T, Ritter H (2010) Approaching manual intelligence. KI-Künstliche Intelligenz 24(4):287-294

22. Musk E Yes, excessive automation at tesla was a mistake. to be precise, my mistake. humans are underrated. [twitter post]. https ://twitter.com/elonmusk/status/984882630947753984. Accessed 19 Apr 2019

23. Pandey AK, Ali M, Alami R (2013) Towards a task-aware proactive sociable robot based on multi-state perspective-taking. Int J Soc Robot 5(2):215-236

24. Patel D, Bhatt J, Trivedi S (2015) Programmable logic controller performance enhancement by field programmable gate array based design. ISA Trans 54:156-168. https://doi.org/10.1016/j. isatra.2014.08.019

25. Pratt GA (2015) Is a cambrian explosion coming for robotics? J Econ Perspect 29(3):51-60

26. Ramirez-Amaro K, Beetz M, Cheng G (2017) Transferring skills to humanoid robots by extracting semantic representations from observations of human activities. Artif Intell 247:95-118

27. Ribeiro L (2017) Cyber-physical production systems' design challenges. Proceedings, 2017 IEEE international symposium on industrial electronics (ISIE). IEEE, Piscataway, pp 1189-1194. https://doi.org/10.1109/ISIE.2017.8001414

28. Russakovsky O, Deng J, Su H, Krause J, Satheesh S, Ma S, Huang Z, Karpathy A, Khosla A, Bernstein M et al (2015) Imagenet large scale visual recognition challenge. Int $\mathrm{J}$ Comput Vis 115(3):211-252

29. Sisbot EA, Alami R (2012) A human-aware manipulation planner. IEEE Trans Robot 28(5):1045-1057

30. SPARC Robotics: Robotics 2020 multi-annual roadmap for robotics in Europe. 2016. https://www.eu-robotics.net/cms/upload/ downloads/ppp-documents/Multi-Annual_Roadmap2020_ICT24_Rev_B_full.pdf. Accessed 19 Apr 2019

31. Suh SH, Kang SK, Chung DH, Stroud I (2008) Programmable logic control. Theory and design of CNC systems, springer series in advanced manufacturing. Springer, London, pp 229-269. https ://doi.org/10.1007/978-1-84800-336-1_7

32. Tenorth M, Beetz M (2013) Knowrob: a knowledge processing infrastructure for cognition-enabled robots. Int J Robot Res 32(5):566-590

33. Thomesse JP (2005) Fieldbus technology and industrial automation. 2005 IEEE conference on emerging technologies and factory automation, vol 1. IEEE, Piscataway, pp 651-653

34. Vogel-Heuser B, Feldmann S, Werner T, Diedrich C (2011) Modeling network architecture and time behavior of distributed control systems in industrial plant automation. Proc IECON 2011. IEEE, Piscataway, pp 2232-2237. https://doi.org/10.1109/IECON .2011 .6119656

35. Vogel-Heuser B, Ribeiro L (2018) Bringing automated intelligence to cyber-physical production systems in factory automation. In: Vogel-Heuser B (ed) 2018 IEEE 14th international conference on automation science and engineering (CASE). IEEE, Piscataway, pp 347-352. https://doi.org/10.1109/COASE.2018.8560430

36. Waibel M, Beetz M, Civera J, d'Andrea R, Elfring J, Galvez-Lopez D, Häussermann K, Janssen R, Montiel J, Perzylo A et al (2011) Roboearth-a world wide web for robots. IEEE RAM Spec Issue Towards WWW Robots 18(2):69-82

37. Xu X, Xiong Z, Wu J, Zhu X (2013) High-precision time synchronization in real-time ethernet-based cnc systems. Int J Adv Manuf Technol 65(5):1157-1170. https://doi.org/10.1007/s0017 0-012-4246-5 